\title{
Polymyositis with Atypical Pathological Features Associated with Thymic Carcinoma
}

\author{
Yumiko Azuma, Kensuke Shiga, Ryotaro Ishii, Tatsuyuki Yamaguchi, Fumitoshi Niwa \\ and Masanori Nakagawa
}

\begin{abstract}
A 66-year-old man was admitted for progressive proximal weakness and myalgia in his shoulder girdles without skin lesions. A muscle biopsy showed infiltration of inflammatory cells, degeneration of muscle fibers, and perifascicular atrophy. Remarkably, MHC-I antigen was expressed in the muscle membrane and most of the inflammatory cells were CD8-positive, suggesting that class-I antigen-dependent cytotoxic T-cells played a crucial role in the muscle pathology, which supported a diagnosis of polymyositis rather than dermatomyositis. Magnetic resonance imaging of his chest revealed two mediastinal tumors, which were extirpated and diagnosed as thymic carcinoma. The muscle weakness was completely recovered after the operation and subsequent administration of oral prednisolone. Postoperative clinical improvement and decline of serum creatinine kinase suggested a paraneoplastic nature of the polymyositis in this patient.
\end{abstract}

Key words: polymyositis, thymic carcinoma, extended thymectomy, steroid, perifascicular atrophy

(Inter Med 48: 163-168, 2009)

(DOI: 10.2169/internalmedicine.48.1136)

\section{Introduction}

Thymic carcinoma is a relatively rare mediastinal tumor of epithelial origin that is distinct from thymoma in terms of its histopathological properties including nuclear atypia and the potential for metastasis $(1,2)$. There have been some case reports of thymic carcinoma complicated with inflammatory myopathies, however, such cases have almost always occurred with dermatomyositis (3-9), wherein the direct link between malignancy and dermatomyositis has been addressed.

Dermatomyositis, polymyositis, and inclusion-body myositis are three distinct classes of inflammatory myopathies, each of which has unique clinical, histopathological, and immunological properties. Dermatomyositis results from a microangiopathy affecting skin and skeletal muscle, wherein activation of complement leads to lysis of endomysial capillaries and muscle ischemia (10), frequently giving rise to perifascicular atrophy. In contrast, in polymyositis and inclusion-body myositis, clonally expanded CD8positive cytotoxic $\mathrm{T}$ cells invade muscle fibers that express
MHC class I antigens $(11,12)$, leading to necrosis of muscle fibers via the perforin pathway. From the clinical aspect, approximately thirty percent of patients with dermatomyositis harbor malignant disorders $(13,14)$, while in polymyositis the link between myopathy and malignancy has often been disputable.

Here we present a patient with polymyositis complicated with thymic carcinoma. Of interest, the muscle biopsy showed fiber atrophy in a perifascicular fashion, however, further immunohistochemical analysis revealed expression of MHC class I antigen on the muscle membrane and infiltration of CD8-positive cells in the endomysial space. Here, we discuss the unique combination of these pathological features and the clinical correlation between malignancy and muscle pathology in this case.

\section{Case Report}

A 66-year-old man was admitted to our hospital for progressive muscle weakness and myalgia in his shoulder girdles. He had been well until five weeks prior to admission when he found it difficult to raise storm shutters with his 

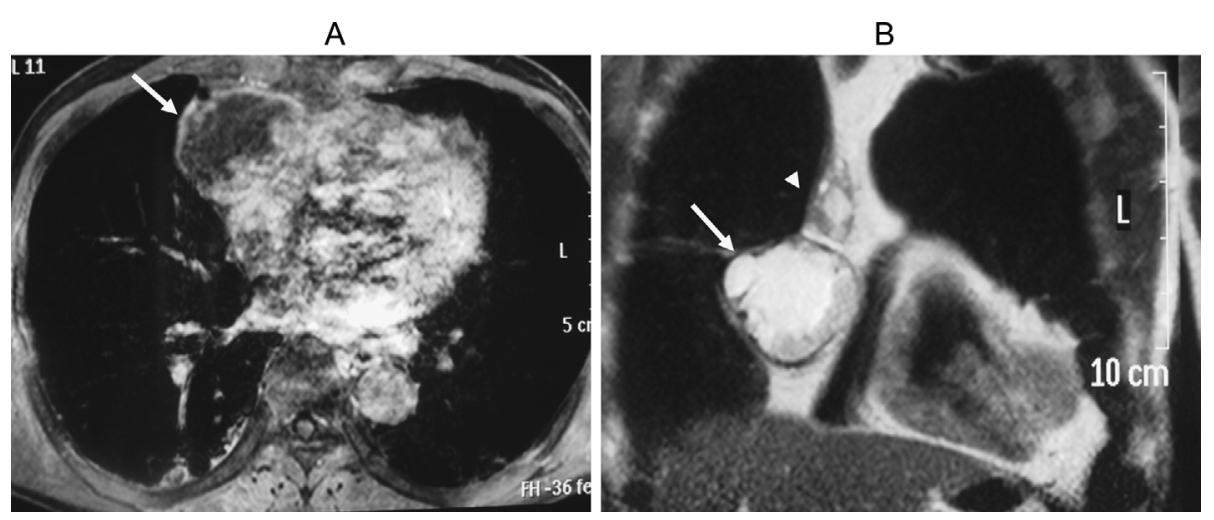

Figure 1. Magnetic resonance images of the chest. A: Axial plane of enhanced image after gadolinium administration. The arrow indicates an anterior mediastinal tumor with an irregular tumor wall protruding inward and containing a low-intensity inside. B: Coronal plane of the T2-weighted image. The arrow indicates the main nodule and the arrowhead shows a daughter nodule above the main nodule.

arms. One week later, he started to have difficulty walking up stairs and unscrewing bottle tops with his hands. Three weeks later myalgia gradually developed in his shoulder girdles. He was then referred to our department. He had a history of type $\mathrm{C}$ chronic hepatitis and bronchial asthma, both of which had been controlled by administration of ursodeoxycholic acid and inhalation of fluticasone propionate, respectively. On examination, his blood pressure was 120/60 $\mathrm{mmHg}$ and his heart rate was 90 beats per minute. His body temperature was $37.5 \mathrm{C}$. Coarse crackles were audible at the base of his lungs, while no rash was noted on his face and extremities including knuckles and patella during a careful inspection by a dermatologist. On neurological examination, Medical Research Council (MRC) scores were 4 in his bilateral deltoid, pectoralis major, supraspinatus, and wrist flexor muscles, and 3 in his infraspinatus and iliopsoas muscles. He also complained of mild myalgia in his shoulder girdles. Other neurological assessments were normal, including those of his cranial nerves, sensory function, and tendon reflexes.

His peripheral white blood-cell count was slightly elevated $\left(11,000 / \mathrm{mm}^{3}\right)$, and the results of his blood chemistry were as follows: lactate dehydrogenase (LDH) 1,168 IU/L, aspartate aminotransferase (AST) 430 IU/L, alanine aminotransferase (ALT) $496 \mathrm{IU} / \mathrm{L}$, creatinine kinase (CK) 7,517 IU/L, and aldolase $200 \mathrm{IU} / \mathrm{L}$. The value of his serum anti-Jo 1 antibody was markedly increased to $390 \mathrm{U} / \mathrm{mL}$ (normal range $<10 \mathrm{U} / \mathrm{mL}$ ), whereas that of his serum Mi-2 antibody was within the normal range. Other myositisspecific antibodies were not investigated.

A needle electromyography in the right deltoid muscle revealed that most of the patient's voluntary muscle activities were comprised of motor units with short duration and low amplitude. Early recruitment was also observed with minimal muscle contraction. Fibrillation potentials and positive sharp waves were frequently recorded at rest. These findings were summarized as myogenic changes with concomitant active denervation. The repetitive supramaximal stimulation test showed no waning response in the left trapezius muscle with $3 \mathrm{~Hz}$ stimulation. Tensilon test was also negative.

A chest X-ray revealed a space-occupying legion in the patient's mediastinum, which was later discerned as a wellcircumscribed irregular nodule (size: $55 \times 45 \mathrm{~mm}$ ) by chest magnetic resonance imaging (MRI) together with an additional daughter nodule located above the main nodule (Fig. 1A, B). The main nodule was comprised of an irregular tumor wall protruding inward and low-intensity substance inside.

A muscle biopsy was performed in the patient's left biceps brachii. The Hematoxylin and Eosin staining section revealed a moderate variation in fiber size, including perifascicular atrophy (Fig. 2A). Numerous necrotic and basophilic fibers were noted and myophagia was also seen in places. Inflammatory cells infiltrated into perimysium and endomysium, but not into endomysial vessels. C5b9, a pathological hallmark of membrane attack complex, was expressed in the muscle membrane in the perifascicular area as well as in degenerating muscle fibers (Fig. 2B), but not around endomysial vessels. Unexpectedly, MHC class I antigen was extensively expressed on most of the muscle membrane (Fig. 3C). In addition, more than half of the inflammatory cells in the endomysium were CD8-positive and CD4negative (Fig. 3A, B). These immunological features and the lack of dermatological presentation led us to diagnose him as having polymyositis rather than dermatomyositis.

The possibility of a paraneoplastic myopathy was raised, which prodded us to extirpate the tumors before administration of a corticosteroid. The two mediastinal tumors were surgically resected by extended thymectomy. Macroscopically, tumors were filled with chocolate-colored necrotic liquid and were encapsulated with a fibrous membrane (Fig. 4A). The tumor was diagnosed as thymic carcinoma that was comprised of three distinct neoplastic components; squamous cell carcinoma, adenocarcinoma, and basaloid cell carcinoma (Fig. 4B, C, and D, respectively). Muscle strength in the deltoid and iliopsoas muscles was gradually improved and the value of serum $\mathrm{CK}$ was decreased to 

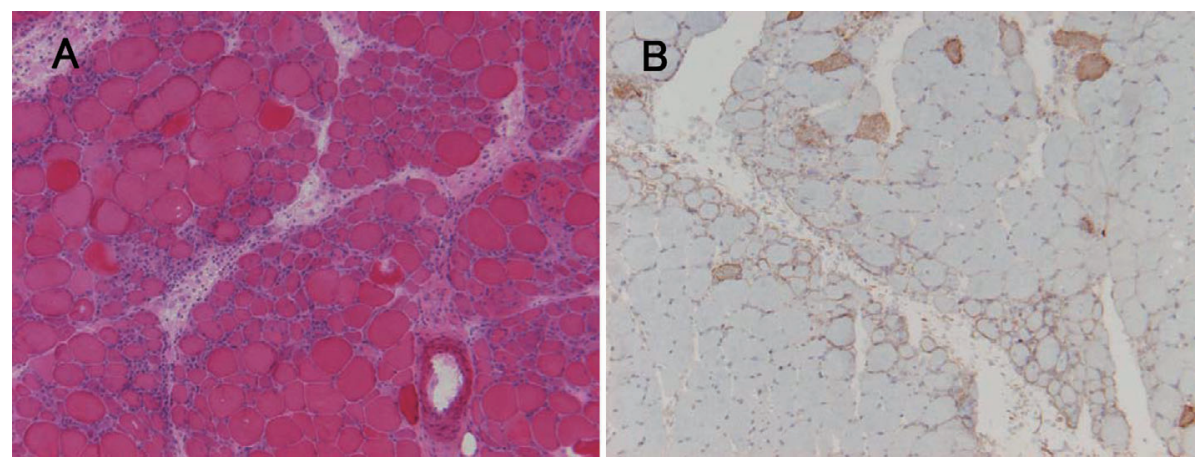

Figure 2. Muscle biopsy specimen from the left deltoid muscle. A: A Hematoxylin and Eosin stained section revealed interstitial edema, infiltration of inflammatory cells, degeneration of muscle fibers, and perifascicular atrophy. B: Immunohistochemistry using the anti-C5b9 antibody showed positive immunostaining of $\mathrm{C5b9}$ in the muscle membrane in the perifascicular area as well as in some degenerating fibers. Scale bar=50 micrometers.
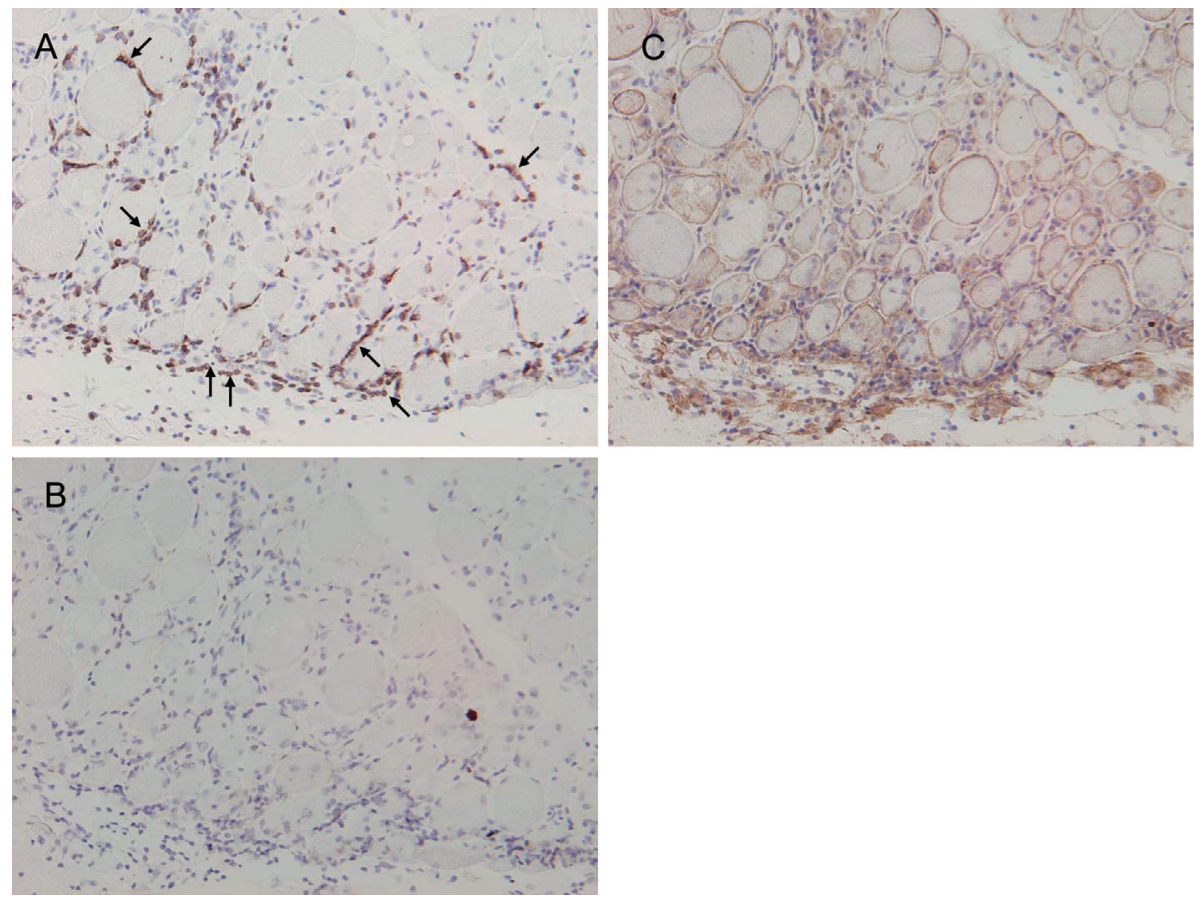

Figure 3. Immunological features in muscle pathology. A: anti-CD8; arrows indicate CD8-positive lymphocytes. B: anti-CD4; no CD4-positive cells were noted. C: anti-MHC-I. The MHC-I was expressed on all muscle fibers $(\mathrm{C})$, and most of the inflammatory cells invading the fibers were CD8 positive (A) and CD4 negative (B).

1,727 IU/L on the fourth day after the operation (Fig. 5). The serum CK level and the muscle strength then reached a plateau on the tenth postoperative day when oral administration of prednisolone was initiated at the dose of $50 \mathrm{mg}$ per day. The muscle strength and the serum CK value subsequently improved, both of which completely normalized in two months. The value of serum anti-Jo 1 antibody also decreased to $10 \mathrm{U} / \mathrm{mL}$ six months after the operation. Interestingly, in retrospect, the value of serum LDH was $217 \mathrm{IU} / \mathrm{L}$ (normal <243 IU/L) two months before the presentation of muscle weakness, implying that there was probably no discernible muscle pathology at that time point.

\section{Discussion}

An increased relative risk for malignant tumors has been reported in patients with inflammatory myopathies, especially those with dermatomyositis $(15,16)$. The complication of cancers in dermatomyositis has been estimated to be approximately $30 \%$ in two independent surveys $(13,14)$, whereas the co-occurrence of cancers in polymyositis has sometimes been challenged. For instance, one nation-wide survey in Japan suggested that the association between polymyositis and cancers may be ten times less frequent than that between dermatomyositis and cancers (17). 

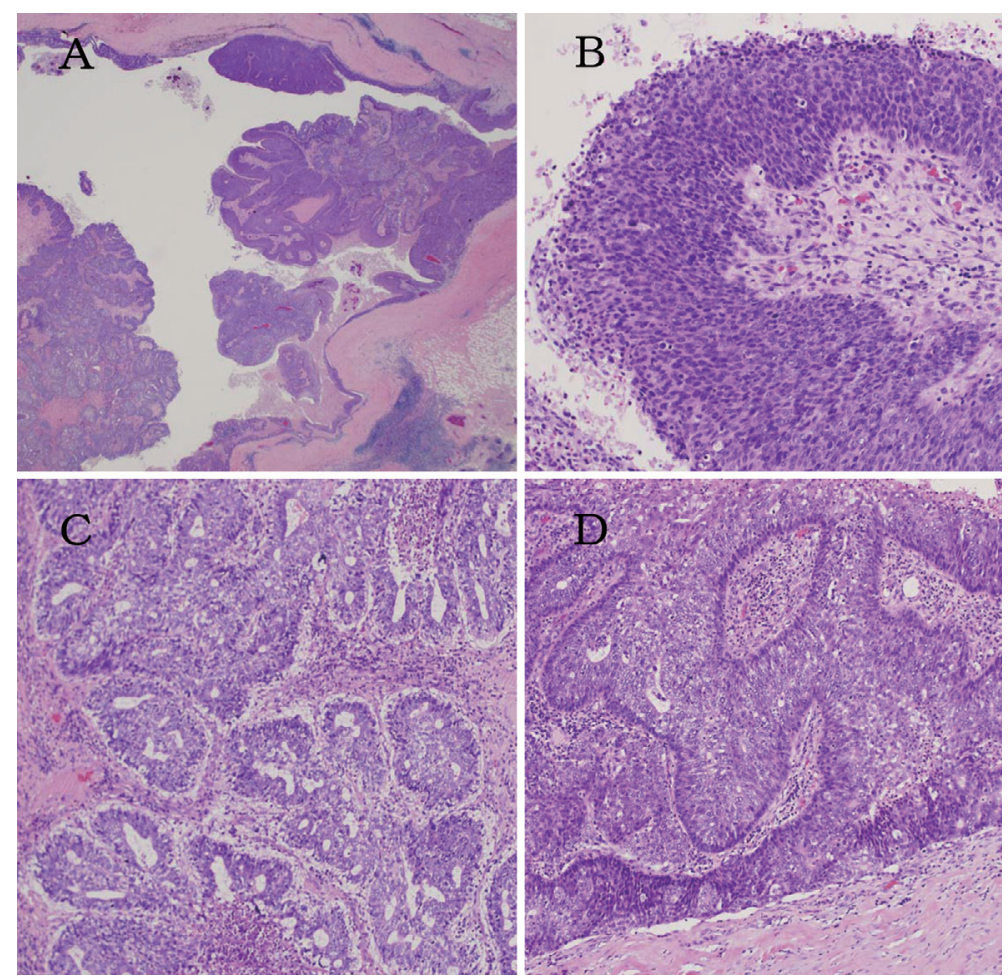

Figure 4. Surgically-resected specimen of the thymic tumor. A: A macroscopic section of the mediastinal tumor. The Hematoxylin and Eosin staining section included the following three different histopathologies (B-D): B: squamous-cell carcinoma, C: adenocarcinoma, and D: basaloid-cell carcinoma.

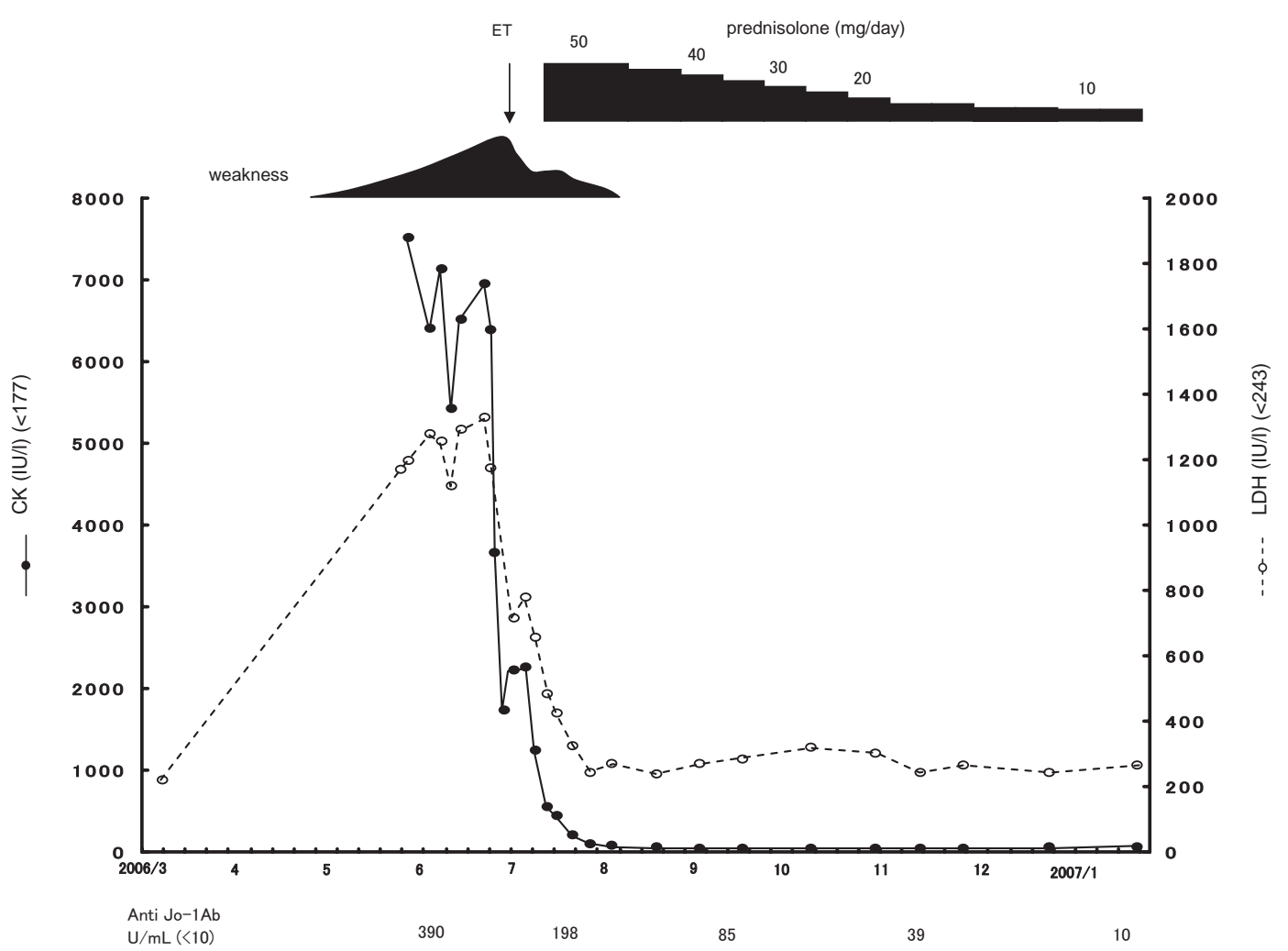

Figure 5. Clinical course of the patient. The serum value of creatine kinase (CK) and lactate dehydrogenase (LDH) decreased after the extended thymectomy and the subsequent administration of oral prednisolone. In retrospect, the serum LDH was within normal range two months before the presentation of the muscle weakness. ET=extend 
Here, we reported a patient with polymyositis who had a concomitant thymic carcinoma. A possible causal relationship between the two conditions is suggested in this case for the following two reasons. First, by simply extirpating the thymic carcinoma, the value of serum CK decreased from 7,517 to 1,727 IU/L and his muscle strength has substantially improved from MRC score 3 to 4 in his iliopsoas muscles (Fig. 5), although he needed subsequent administration of oral prednisolone for full recovery. Second, the serum value of $\mathrm{LDH}$, another indicator of muscle injury, was within the normal range two months before the presentation of the muscle symptoms (Fig. 5), suggesting the pathologic process in the skeletal muscles had subsequently started probably as the thymic carcinoma increased in size. The recent onset of muscle pathology and the favorable response of tumor resection for polymyositis indicate that the paraneoplastic mechanism has probably influenced the development of muscle pathology in this patient.

A unique characteristic of the muscle pathology in this case was an unexpected combination of perifascicular atrophy (Fig. 2A) and the expression of the CD8/MHC-1 complex (Fig. 3A, C). The former finding has been generally regarded as a morphological consequence of endomysial microangitis resulting from complement activation (10), rendering dermatomyositis as a possible diagnosis. The latter immunological findings, clearly indicated that class-I antigen-dependent cytotoxic $\mathrm{T}$ cells played a crucial role in its pathogenesis $(11,12)$, suggesting polymyositis as a reasonable diagnosis. Therefore, we infer that polymyositis would be a more plausible diagnosis for this patient for the following two reasons.

First, the current working diagnostic criteria for inflammatory myopathies stipulate that primary inflammation by the CD8/MHC-1 complex and absence of a skin rash lead to the diagnosis of "definite" polymyosits (18). Secondly, our case lacked two cardinal features of dermatomyositis, i.e., morphological evidence of angitis and invasion of CD4positive $\mathrm{T}$ cells. In addition, careful examination by a dermatologist did not reveal any skin rash in this patient. We thus concluded that dermatomyositis would be a less plausible diagnosis in our case, although the muscle pathology had two atypical features of polymyositis, i.e., perifascicular atrophy and complement activation.

First, we theorize that the perifascicular atrophy seen in our case probably did not represent microangitis, the putative cause of perifascicular atrophy; firstly because inflammatory cells infiltrated primarily into endomysium and perimysium but not around the vasculature; secondly because $\mathrm{C}$ $5 \mathrm{~b} 9$, the membrane attack complex (MAC), was deposited mainly in degenerating muscle fibers along the perifascicular areas and not in the capillaries. It is true that perifascicular atrophy is observed more frequently in patients with dermatomyositis (19), however, seven percent of patients with polymyositis have been reported to have typical perifascicular atrophy (20). Of note, another study showed that approximately thirty percent of patients with polymyositis and concomitant neoplasm have perifascicular fiber atrophy (21), which may suggest a possible link between malignant tumors and the morphological changes in this class of patients, although the mechanisms remain to be elucidated.

Second, activation of the complement system seen in our case (Fig. 2B) is not a typical finding in polymyositis, however, MAC deposition in muscle fibers has been reported in a portion of inflammatory myopathies, in fifty percent of the cases of fascioscapulohumeral dystrophy and merosinpositive congenital muscular dystrophy (22). We figure that complement activation in muscle fibers may represent nonspecific findings of muscle-fiber injury in various classes of myopathies, including muscular dystrophies and inflammatory myopathies.

Thymic carcinoma is a relatively rare mediastinal carcinoma of epithelial origin that is distinct from thymoma in the following aspects: 1) it has a cellular atypia, 2) it has lost its capacity to induce lymphocytes, and 3) it can metastasize to remote regions $(1,2)$. To date, there have been eight cases with thymic carcinoma that were complicated with inflammatory myopathies, however, seven such cases had dermatomyositis (3-9) and only one patient had polymyositis (23); the latter case being diagnosed at autopsy. At the other end of the spectrum, there have been many case reports of patients with polymyositis complicated with thymoma (24). In relation to collagen diseases, thirty to forty percent of patients with thymoma have myasthenia gravis, five to ten percent involve erythropenia, five percent have polymyositis (24), one percent have Hashimoto thyroiditis, and 0.9 percent have Sjögren syndrome (25). Of interest, the patients with thymic carcinoma and inflammatory myopathies were also complicated with other immunological diseases, such as Hashimoto thyroiditis and Sjögren syndrome (4). The thymus is a central immunological organ for immune tolerance, playing a critical role in T-cell maturation and its apoptotic pathways. The perturbation of this immunological center, either benign or malignant, may result in various collagen-vascular diseases including polymyositis. Atypical morphological and immunological features in the muscle pathology may reflect that perturbation of the immune system to some extent.

In summary, we report a patient with polymyositis complicated with thymic carcinoma, whose serum CK value and muscle weakness were substantially improved by simply extirpating the mediastinal tumors. The favorable response to the surgical procedure and the perifascicular muscle pathology sometimes observed in polymyositis with malignancy may suggest a paraneoplastic etiology of polymyositis in this patient. 


\section{References}

1. Shimosato Y, Kayama T, Nagai K, Suemasu K. Squamous cell carcinoma of the thymus. An analysis of eight cases. Am J Surg Pathol 1: 109-121, 1977.

2. Yokoi K, Usami N, Uchiyama M. Thymoma and thymic cancer. Geka Chiryo 96: 354-358, 2007 (in Japanese).

3. Fong PH, Wee A, Chan HL, Tan YO. Primary thymic carcinoma and its association with dermatomyositis and pure red cell aplasia. Int J Dermatol 31: 426-428, 1992.

4. Kurisu S, Nagata H, Yokote K, Yamate N. Thymic carcinoma associated with Sjögren syndrome, Hashimoto's disease and dermatomyositis; a case report. Nippon Kokyuki Geka Gakkai Zasshi 8: 52-55, 1994 (in Japanese, Abstract in English).

5. Ochiai Y, Fujita Y, Uehara T, et al. A case of dermatomyositis complicated with thymic carcinoma. Nippon Hifuka Gakkai Zasshi 110: 200, 2002 (in Japanese) (Abstract).

6. Kogure Y, Numao T, Okada S, et al. A case of dermatomyositis complicated with thymic carcinoma. Ryumachi 38: 246, 1998 (in Japanese) (Abstract).

7. Kagaya M, Saga K, Kamada A, et al. Dermatomyositis associated with thymic carcinoma. Rinsho Hifuka 58: 441-444, 2004 (in Japanese).

8. Saito Y, Miyamoto H, Sakao Y, et al. A resection case of dermatomyositis complicated with thymic carcinoma. Proceedings of the Japanese Lung Cancer Society Lung Cancer 45: 200, 2005 (in Japanese) (Abstract)

9. Sato F, Kato N, Sasamoto S, et al. A case of dermatomyositis complicated with thymic carcinoma. Nippon Kyobu Geka Gakkai Zasshi, The 134th Japanese Association for Thoracic Surgery, Kanto-Ko-Shin-Etsu Chapter 25, 2005 (in Japanese) (Abstract).

10. Kissel JT, Mendell JR, Rammohan KW. Microvascular deposition of complement membrane attack complex in dermatomyositis. New Engl J Med 314: 329-334, 1986.

11. Karpati G, Pouliot Y, Carpenter S. Expression of immunoreactive major histocompatibility complex products in human skeletal muscles. Ann Neurol 23: 64-72, 1988.

12. Arahata K, Engel AG. Monoclonal antibody analysis of mononuclear cells in myopathies. III: Immunoelectron microscopy aspects of cell-mediated muscle fiber injury. Ann Neurol 19: 112-125, 1986.
13. Hidano A, Kaneko K, Arai Y, Kikuchi R. Survey of the prognosis for dermatomyositis, with special reference to its association with malignancy and pulmonary fibrosis. J Dermatol 13: 233-241, 1986.

14. Bonnetblanc JM, Bernard P, Favor J. Dermatomyositis and malignancy. A multicenter cooperative study. Dermatologica 180: 212216, 1990.

15. Sigurgeirsson B, Lindelöf B, Edhag D, Allander E. Risk of cancer in patients with dermatomyositis or polymyositis. A population based-study. N Engl J Med 326: 363-367, 1992.

16. Zantos D, Zhang Y, Felson D. The overall and temporal association of cancer with polymyositis and dermatomyositis. J Rheumatol 21: 1855-1859, 1994.

17. Shinojima H, Nonami E, Ikegami F, Takasu Y, Takei H, Saito H. Dermatomyositis complicated with malignancy. Hifuka No Rinsho 19: 743-752, 1977 (in Japanese).

18. Dalakas MC, Hohlfeld R. Polymyositis and dermatomyositis. The Lancet 362: 971-982, 2003.

19. Engel, Andrew G, Clara Franzini-Armstrong. Myology. 3rd ed. McGraw-Hill, New York, 2004: 724.

20. Serratrice G, Schiano A, Pellissier JF, Pouget J. 135 cases of polymyositis. Rev Neurol (Paris) 142: 906-917, 1986 (in French, Abstract in English).

21. Peiffer J, Bähr M. Anomalies in perifascicular muscle fibers as a differential diagnostic criterion. I.Perifascicular atrophy in inflammatory myopathies. Clin Neuropathol 6: 123-132, 1987.

22. Spuler S, Engel AG. Unexpected sarcolemmal complement membrane attack complex deposits on nonnecrotic muscle fibers in muscular dystrophies. Neurology 50: 41-46, 1998.

23. Le Marc'hadour F, Martins Ramos J, Pasquier B, Pasquier D, Couderc P. Association of thymus carcinoma, Hashimoto's thyroiditis and polymyositis. Anatomoclinical case with autopsy findings. Ann Pathol 9: 355-359, 1989 (in French, Abstract in English).

24. Ago T, Nakamura M, Iwata I, et al. Dermatomyositis associated with invasive thymoma. Intern Med 38: 155-159, 1999.

25. Souadjian JV, Enriquez P, Silverstein MN, Pepin JM, Minn R. The spectrum of diseases associated with thymoma. Coincidence or syndrome? Arch Intern Med 134: 374-379, 1974.

(C) 2009 The Japanese Society of Internal Medicine http://www.naika.or.jp/imindex.html 